\title{
Intraocular pressure readings obtained through soft contact lenses using four types of tonometer
}

This article was published in the following Dove Press journal:

Clinical Ophthalmology

5 October 2015

Number of times this article has been viewed

\author{
Joji Takenaka' \\ Eriko Kunihara' \\ Ulfah Rimayanti ${ }^{2}$ \\ Junko Tanaka ${ }^{3}$ \\ Makoto Kaneko 4 \\ Yoshiaki Kiuchi' \\ 'Department of Ophthalmology and \\ Visual Science, Graduate School of \\ Biomedical Sciences, Hiroshima \\ University, Hiroshima, Japan; ${ }^{2}$ Faculty \\ of Medicine and Health Sciences, \\ UIN Alauddin Makassar, South \\ Sulawesi, Indonesia; ${ }^{3}$ Department \\ of Epidemiology, Infectious Disease \\ Control and Prevention, Graduate \\ School of Biomedical Sciences, \\ Hiroshima University, Hiroshima, \\ Japan; ${ }^{4}$ Graduate School of \\ Engineering, Osaka University, Osaka, \\ Japan
}

\begin{abstract}
Purpose: To compare the reliability and accuracy of intraocular pressure (IOP) measured while wearing soft contact lenses (SCLs) using a non-contact tonometer (NCT), Goldmann applanation tonometer (GAT), iCare rebound tonometer (RBT) and the Tono-Pen XL.

Methods: Twenty-six healthy subjects were examined. The IOP was measured using NCT, GAT, RBT, and the Tono-Pen XL, while the subjects wore SCLs $-5.00 \mathrm{D},-0.50 \mathrm{D}$ and +5.00 D. Bland-Altman plots and a regression analysis were used to compare the IOPs obtained with those instruments and the IOPs of the naked eyes measured using GAT (the standard IOPs in this study).

Results: The IOPs obtained by the Tono-Pen XL while the subjects were wearing $-5.00 \mathrm{D},-0.50$ $\mathrm{D}$, and +5.00 D SCLs were significantly higher than those of the naked eyes obtained using GAT. RBT showed that the IOPs were similar to the GAT standard IOPs under all conditions. The IOPs measured with NCT and GAT while the subjects were wearing $-5.00 \mathrm{D}$ and $-0.50 \mathrm{D}$ SCLs were similar to the GAT standard IOPs. The IOPs obtained with RBT and NCT while the subjects were wearing $-5.00 \mathrm{D}$ and $-0.50 \mathrm{D}$ SCLs exhibited a good correlation with the standard IOPs.

Conclusion: The NCT and RBT are best when measuring IOP through hydrogel SCLs.

Keywords: soft contact lens, intraocular pressure, rebound tonometer, non-contact tonometer
\end{abstract}

\section{Introduction}

Soft contact lenses (SCLs) are often used in the treatment of corneal epithelial abnormalities and after corneal surgery. Contact lenses (CLs) are also a common tool for correcting refractive errors. The intraocular pressure (IOP) is a fundamental and essential ocular parameter in ophthalmological clinics. Physicians treating corneal disease and refractive errors need to estimate whether the IOP is beyond the normal limit. Measuring the IOP through SCLs is convenient for ophthalmologists treating corneal and refractive diseases. However, it has been shown that wearing CLs during IOP measurement affects the results. ${ }^{1-3}$ Previous studies have reported IOP changes using different instruments. ${ }^{1-10}$ For example, when using a non-contact tonometer (NCT), a significant increase in the IOP was found when subjects used plus SCLs, while a decrease was observed when the subjects used minus SCLs made of hydrogel and silicone hydrogel. ${ }^{1,2}$ With respect to Goldmann applanation tonometer (GAT) measurement, neither $-1.50 \mathrm{D}$ hydrogel $\mathrm{SCLs}^{5}$ nor $-0.50 \mathrm{D}$ silicone hydrogel $\mathrm{SCLs}^{6}$ have significant effects on IOP results. Furthermore, studies have reported that the use of SCLs for therapy does not affect the IOP obtained using the Tono-Pen XL, ${ }^{7,8}$ whereas Zeri et $\mathrm{al}^{9}$ reported that the iCare rebound tonometer (RBT) shows significant differences in the IOP values among subjects wearing $+2.00 \mathrm{D},-2.00 \mathrm{D}$ and $-6.00 \mathrm{D}$ hydrogel CLs versus those wearing no lenses.
Correspondence: Joji Takenaka Department of Ophthalmology and Visual Science, Graduate School of Biomedical Sciences, Hiroshima University, I-2-3 Kasumi, Minami-ku, Hiroshima 734-855I, Japan

Tel +8I 822575247

Fax +81822575249

Email george-00I@hiroshima-u.ac.jp 
However, there have been no comparisons of the IOP measurements obtained using the NCT, GAT, RBT or Tono-Pen XL while wearing SCLs. In order to clarify which tonometer is more reliable for measuring the IOP through SCLs, we examined the IOP results obtained while wearing SCLs using these instruments.

\section{Methods}

The right eye in each of 26 healthy subjects was examined between August and December 2009. The procedures adhered to the tenets of the Declaration of Helsinki, and were approved by the Institutional Review Board of Hiroshima University, Japan. Informed consent was obtained from each subject after informing them of the nature and possible complications of the examination procedures. Subjects with a history of ocular surgery or any type of eye disease other than refractive errors were excluded. All subjects underwent standard ophthalmological examinations, including slit-lamp examinations and ophthalmoscopy. The corneal central thickness was measured with a specular microscope (SP-2000p; Topcon Corporation, Tokyo, Japan). The refractive error (spherical equivalent) and corneal curvature were measured with an autorefractor/autokeratometer (ARK-700A; NIDEK Co. Ltd., Gamagori, Japan).

All IOP-measuring instruments were calibrated prior to the examinations. The IOP values of the naked eyes were examined with NCT (CT-70; Topcon), GAT, RBT (Tiolat Oy, Helsinki, Finland), and Tono-pen XL (Mentor, Norwell, MA, USA) with 5-minute intervals for every measurement in accordance with previous articles. ${ }^{9,11}$ The IOP was then measured while the subject wore hydrogel ACUVUE CLs (Johnson \& Johnson, New Brunswick, NJ, USA) of $-5.00 \mathrm{D},-0.50 \mathrm{D}$ and $+5.00 \mathrm{D}$, with an $8.7 \mathrm{~mm}$ base curve, $14.0 \mathrm{~mm}$ diameter, and $58 \%$ water content. The IOP without a bar display were adopted for the RBT. Further, only readings with a maximum $5 \%$ standard deviation were recorded with the Tono-pen XL. IOP readings that appear in parentheses were excluded when using the NCT. We measured IOP three times using GAT and averaged the IOP readings. We measured the IOP in random order. All examinations were performed by an ophthalmologist between $10 \mathrm{am}$ and $3 \mathrm{pm}$. The sample size was decided as follows. We decided that the statistical power was to be 0.80 . Thus, to calculate the necessary sample size, we presumed that between the tonometers, there was a standard deviation (SD) of $2 \mathrm{mmHg}$, a difference $(\Delta)$ of $2 \mathrm{mmHg}$, a type 1 error $(\alpha)$ of 0.01 , and type 2 error $(\beta)$ of 0.20 . Therefore the necessary sample size $(\mathrm{N})=2(\mathrm{Z} \alpha / 2+\mathrm{Z} \beta)^{2} \mathrm{SD}^{2} / \Delta^{2}=23.4$.

\section{Statistical analyses}

The relationships between the IOP values of the naked eyes measured using GAT and those obtained while wearing SCLs measured with various instruments were evaluated according to Bland-Altman plots and a regression analysis. The results of the regression analysis were interpreted for the Spearman rank correlation coefficient as follows: high correlation $(r=0.7-0.99)$, moderate correlation $(r=0.4-0.69)$, weak correlation $(r=0.2-0.39)$, and no correlation $(r<0.2)$. We regarded the IOP values of the naked eyes measured using GAT to be the standard IOP values in this study. The differences between the average standard IOP values and the IOP values obtained while wearing SCLs measured using various instruments were evaluated using the Student's $t$-test. A $P$-value of $<0.05$ was considered statistically significant. The statistical analyses were performed using the JMP 10.0 version software program (SAS Institute Inc., Cary, NC, USA).

\section{Results}

There were 26 subjects consisting of 15 females and eleven males, with a mean age of $21.9 \pm 1.1$ years. The demographics of subjects are presented in Table 1.

The mean IOP obtained without SCLs using GAT was $13.7 \pm 2.6 \mathrm{mmHg}$. The changes in the IOP values obtained while wearing $-5.00 \mathrm{D},-0.50 \mathrm{D},+5.00 \mathrm{D}$ CLs measured using the various instruments compared to the IOP values of the naked eyes measured using GAT are presented in Table 2. The IOP values measured using $\mathrm{NCT}$ while wearing $-5.00 \mathrm{D}$ and -0.50 D SCLs were not significantly different from the standard IOP values, although the IOP values obtained while wearing +5.00 D lenses were significantly higher $(P<0.01)$ than the standard IOP values. Similar to these results, the IOP values obtained using GAT while the subject was wearing -0.50 D SCLs were not significantly different, whereas those obtained while the subject was wearing $-5.00 \mathrm{D}$ lenses were significantly lower ( $P=0.03)$, and those obtained while the subject was wearing $+5.00 \mathrm{D}$ lenses were significantly higher $(P<0.01)$ than the standard IOP values. The IOP measurements obtained with SCLs using RBT did not show any significant differences

Table I Subject demographics

\begin{tabular}{lll}
\hline Parameter & Average & Range \\
\hline Age, years & $21.9 \pm \mathrm{I} . \mathrm{I}$ & $20-24$ \\
Sex, F/M & $15 / \mathrm{II}$ & - \\
CCT, $\mu \mathrm{m}$ & $536.3 \pm 29.2$ & $484-582$ \\
Corneal curvature, mm & $7.8 \pm 0.2$ & $7.5-8.2$ \\
Spherical equivalent, D & $-4.5 \pm 2.7$ & $-8.8-0$ \\
\hline
\end{tabular}

Note: The values are presented as the mean \pm standard deviation. Abbreviations: F/M, female/male; CCT, corneal central thickness. 
Table 2 Intraocular pressure differences in the subjects wearing soft contact lenses compared to that obtained without contact lenses measured using the Goldmann applanation tonometer

\begin{tabular}{|c|c|c|c|c|c|c|c|c|}
\hline \multirow{2}{*}{$\begin{array}{l}\text { Contact lens } \\
\text { power } \\
\text { (diopter) }\end{array}$} & \multicolumn{2}{|l|}{ NCT } & \multicolumn{2}{|l|}{ GAT } & \multicolumn{2}{|l|}{ RBT } & \multicolumn{2}{|c|}{ Tono-Pen XL } \\
\hline & $\begin{array}{l}\text { Difference } \\
(\mathrm{mmHg})\end{array}$ & $P$-value & $\begin{array}{l}\text { Difference } \\
(\mathrm{mmHg})\end{array}$ & $P$-value & $\begin{array}{l}\text { Difference } \\
\text { (mmHg) }\end{array}$ & $P$-value & $\begin{array}{l}\text { Difference } \\
\text { (mmHg) }\end{array}$ & $P$-value \\
\hline-5.00 & -0.5 & 0.42 & -1.5 & 0.03 & +0.5 & 0.49 & +2.6 & $<0.01$ \\
\hline-0.50 & +0.3 & 0.70 & -0.6 & 0.36 & -0.2 & 0.75 & +2.5 & $<0.01$ \\
\hline+5.00 & +2.0 & $<0.01$ & +2.3 & $<0.01$ & +1.1 & 0.18 & +4.8 & $<0.01$ \\
\hline
\end{tabular}

Abbreviations: NCT, non-contact tonometer; GAT, Goldmann applanation tonometer; RBT, iCare rebound tonometer.

compared to standard IOP measured using GAT. In contrast, all IOP measurements obtained with SCLs using the Tono-Pen XL exhibited significant differences compared to the standard IOP values (all $P<0.01$ ).

The relationships between the standard IOP values and the IOP values obtained while wearing SCLs measured using NCT, GAT, RBT, and the Tono-Pen XL are displayed with Bland-Altman plots in Figures $1-4$, respectively. The $\mathrm{x}$-axis represents the average of the GAT standard IOP and each measurement, while the $y$-axis represents the difference between the two values. The differences in the IOP values measured using NCT while wearing $-5.00 \mathrm{D}$ and $-0.50 \mathrm{D}$ lenses were close to 0 , whereas those obtained while wearing $+5.00 \mathrm{D}$ lenses showed increased IOP measurements of $2.0 \mathrm{mmHg}$. For the $-5.00 \mathrm{D},-0.50 \mathrm{D}$, and $+5.00 \mathrm{D}$ lenses, the $95 \%$ limit of agreement was $-0.5 \pm 3.2 \mathrm{mmHg}$, $0.3 \pm 2.0 \mathrm{mmHg}$, and $2.0 \pm 3.7 \mathrm{mmHg}$, respectively. Meanwhile, the GAT measurements showed decreased values of $1.5 \mathrm{mmHg}$ and $0.6 \mathrm{mmHg}$ while wearing $-5.00 \mathrm{D}$ and -0.50 D CLs, respectively, and an increased value of $2.3 \mathrm{mmHg}$
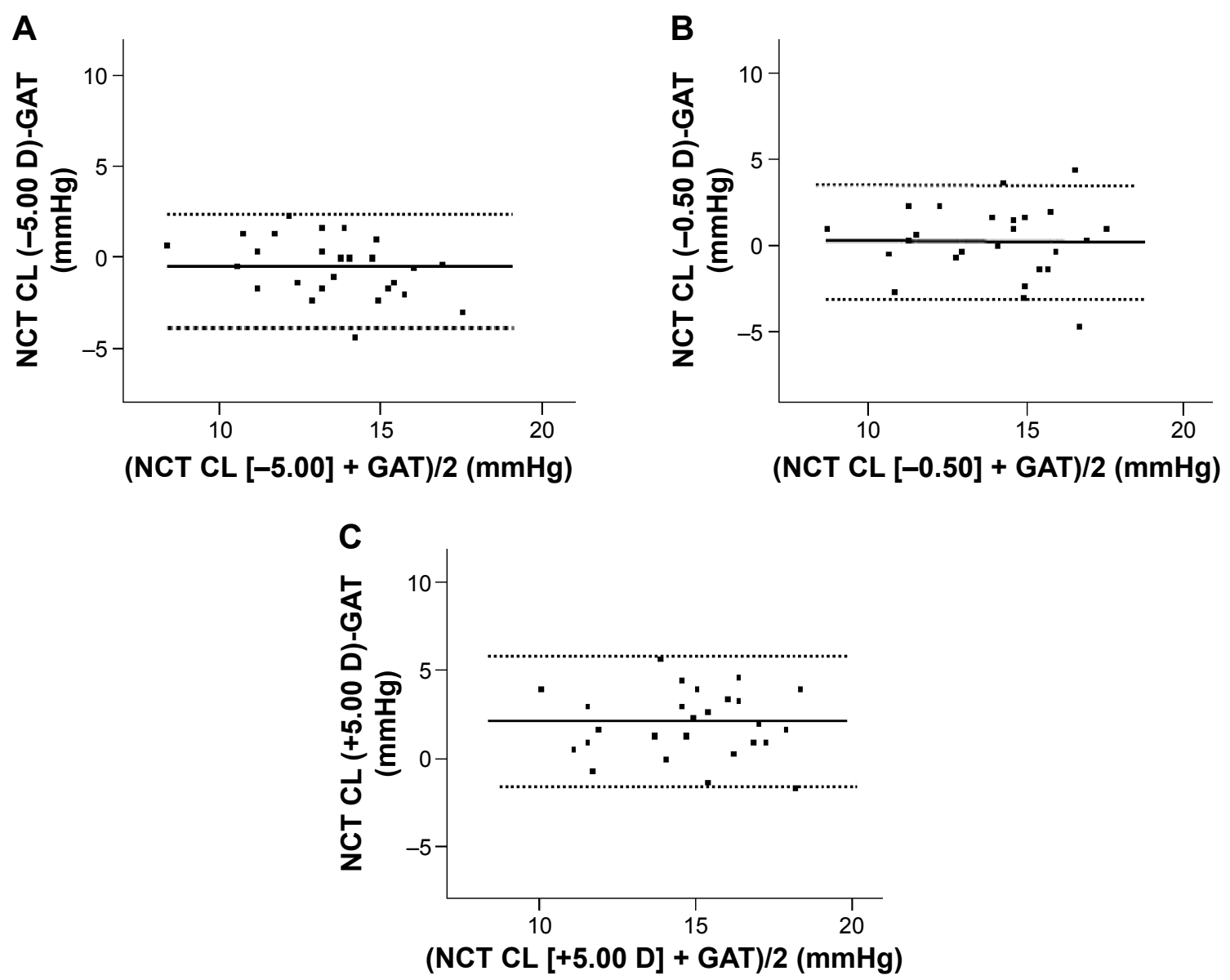

Figure I Bland-Altman plot showing the relationship between the IOP values of the naked eyes measured using GAT and those obtained while wearing SCLs measured using NCT.

Notes: (A) Wearing -5.00 D SCLs. (B) Wearing -0.50 D SCLs. (C) Wearing +5.00 D SCLs.

Abbreviations: IOP, intraocular pressure; GAT, Goldmann applanation tonometer; SCLs, soft contact lenses; NCT, non-contact tonometer; CL, contact lens; D, diopter. 

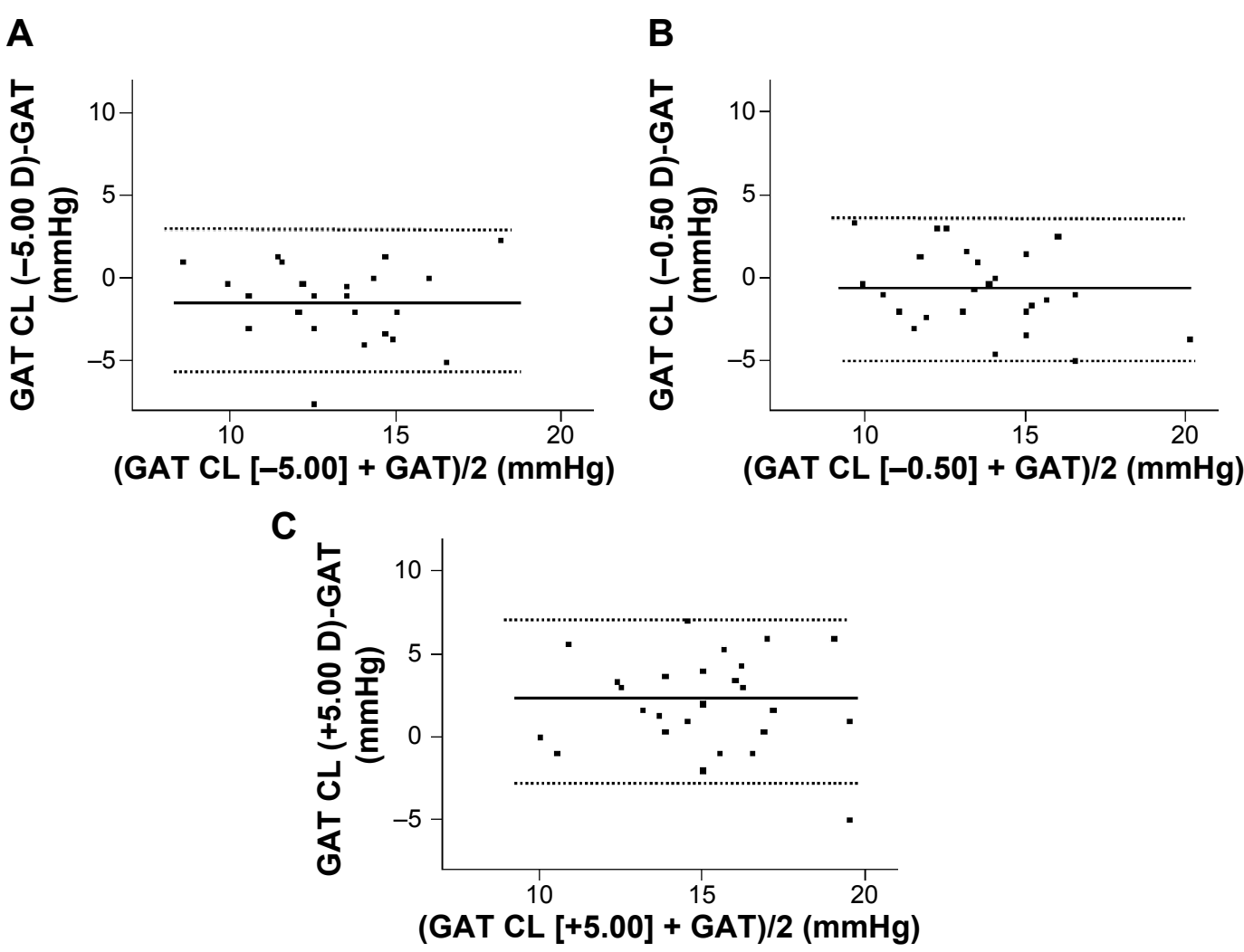

Figure 2 Bland-Altman plot showing the relationship between the IOP values of the naked eyes and those obtained while wearing SCLs measured using GAT. Notes: (A) Wearing -5.00 D SCLs. (B) Wearing -0.50 D SCLs. (C) Wearing +5.00 D SCLs.

Abbreviations: IOP, intraocular pressure; GAT, Goldmann applanation tonometer; SCLs, soft contact lenses; CL, contact lens; D, diopter.
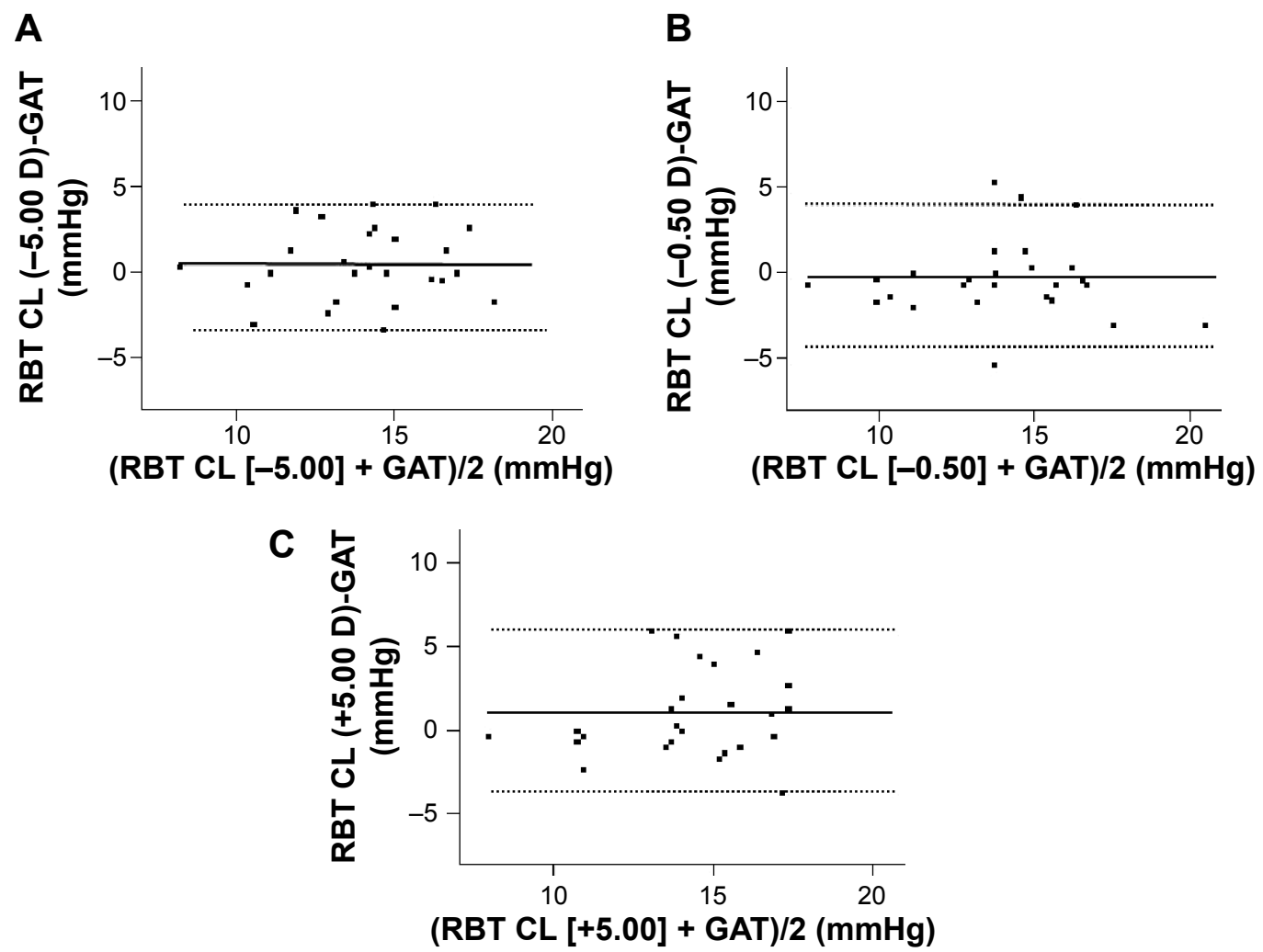

Figure 3 Bland-Altman plot showing the relationship between the IOP values of the naked eyes measured using GAT and those obtained while wearing SCLs measured using RBT. Notes: (A) Wearing -5.00 D SCLs. (B) Wearing -0.50 D SCLs. (C) Wearing +5.00 D SCLs.

Abbreviations: IOP, intraocular pressure; GAT, Goldmann applanation tonometer; SCLs, soft contact lenses; CL, contact lens; D, diopter; RBT, iCare rebound tonometer. 

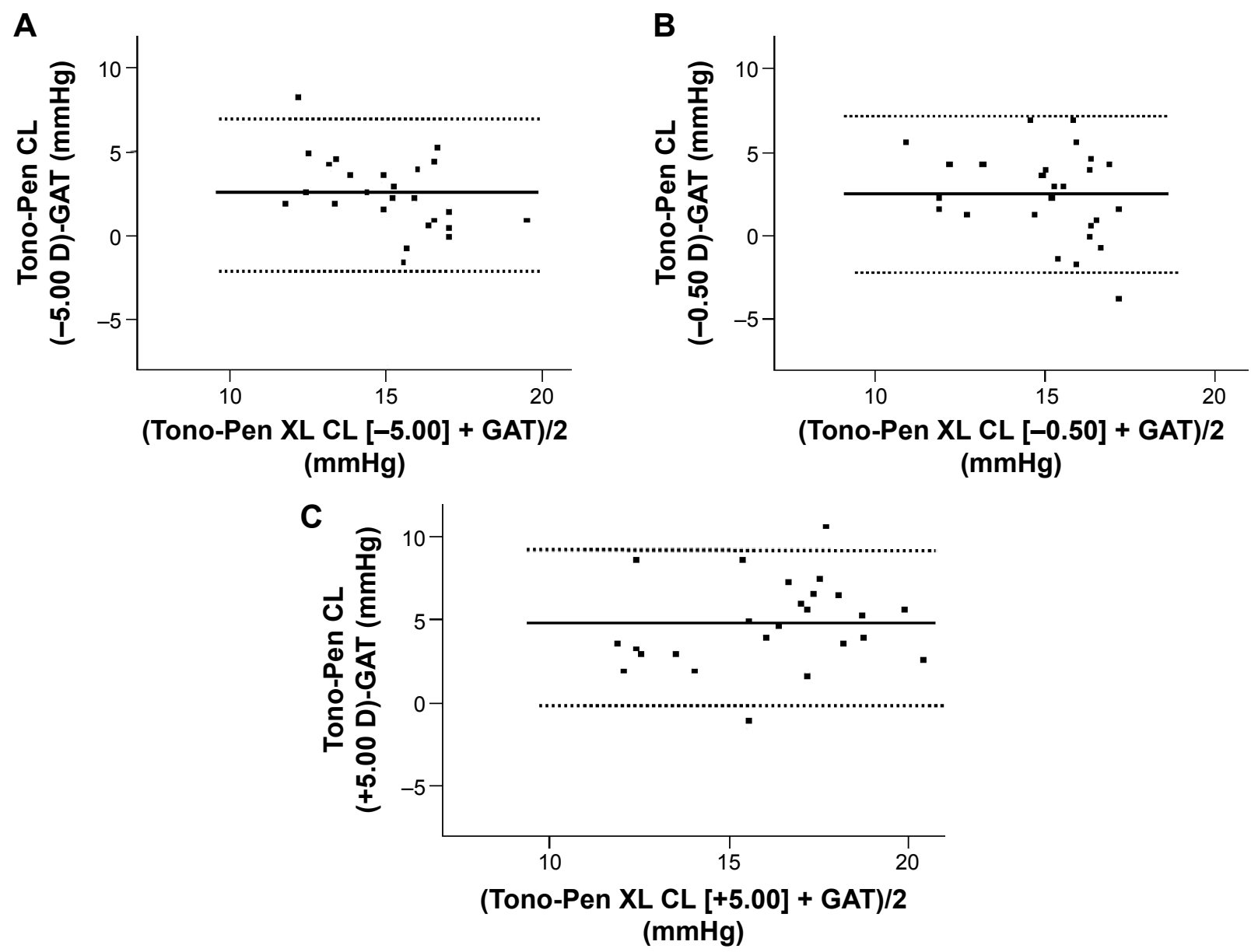

Figure 4 Bland-Altman plot showing the relationship between the IOP values of the naked eyes measured using GAT and those obtained while wearing SCLs measured using the Tono-Pen XL.

Notes: (A) Wearing -5.00 D SCLs. (B) Wearing -0.50 D SCLs. (C) Wearing +5.00 D SCLs.

Abbreviations: IOP, intraocular pressure; GAT, Goldmann applanation tonometer; SCLs, soft contact lenses; CL, contact lens; D, diopter.

while wearing $+5.00 \mathrm{D}$ CLs. The $95 \%$ limit of agreement was $-1.5 \pm 4.4 \mathrm{mmHg},-0.6 \pm 4.4 \mathrm{mmHg}$, and $2.3 \pm 4.8 \mathrm{mmHg}$ for $-5.00 \mathrm{D},-0.50 \mathrm{D}$, and $+5.00 \mathrm{D}$ CLs, respectively. The RBT measurements showed that the differences in IOP were close to 0 while wearing $-5.00 \mathrm{D},-0.50 \mathrm{D}$, and $+5.00 \mathrm{D}$ lenses. The $95 \%$ limit of agreement for the $-5.00 \mathrm{D},-0.50 \mathrm{D}$, and $+5.00 \mathrm{D}$ lenses were $0.5 \pm 4.3 \mathrm{mmHg},-0.2 \pm 4.9 \mathrm{mmHg}$, and $1.1 \pm 5.2 \mathrm{mmHg}$, respectively. The Tono-Pen XL showed higher IOP differences while wearing $-5.00 \mathrm{D},-0.50 \mathrm{D}$, and $+5.00 \mathrm{D}$ lenses, with a $95 \%$ limit of agreement of $2.6 \pm 4.2 \mathrm{mmHg}$, $2.5 \pm 5.6 \mathrm{mmHg}$, and $4.9 \pm 5.2 \mathrm{mmHg}$, respectively.

The results of the regression analyses of the IOP values of the naked eyes measured using GAT and the IOP values obtained while wearing SCLs measured using the various instruments are shown in Table 3. The IOP values measured using NCT showed a moderate correlation with the standard IOP values among the subjects wearing SCLs. Meanwhile, the IOP values measured using RBT exhibited moderate correlations among the subjects wearing $-5.00 \mathrm{D}$ and $-0.50 \mathrm{D}$
Table 3 Results of the regression analysis of the intraocular pressure values of the naked eyes measured using the Goldmann applanation tonometer, and those obtained while wearing contact lenses measured with various tonometers

\begin{tabular}{|c|c|c|c|}
\hline Tonometer & $r$ value & $P$-value & Regression equation \\
\hline NCT & 0.78 & $<0.0001$ & $y=0.72 x+4.47$ \\
\hline NCT CL $-5.00 \mathrm{D}$ & 0.60 & $<0.000 \mathrm{I}$ & $y=0.59 x+5.10$ \\
\hline NCT CL $-0.50 \mathrm{D}$ & 0.44 & 0.0002 & $y=0.64 x+5.22$ \\
\hline NCT CL $+5.00 \mathrm{D}$ & 0.52 & $<0.0001$ & $y=0.70 x+6.08$ \\
\hline GAT CL $-5.00 \mathrm{D}$ & 0.36 & 0.0013 & $y=0.56 x+4.55$ \\
\hline GAT CL $-0.50 \mathrm{D}$ & 0.27 & 0.0060 & $y=0.41 x+7.54$ \\
\hline GAT CL $+5.00 \mathrm{D}$ & 0.34 & 0.0017 & $y=0.65 x+7.15$ \\
\hline RBT & 0.60 & $<0.000$ I & $y=1.00 x+1.47$ \\
\hline RBT CL $-5.00 \mathrm{D}$ & 0.46 & 0.0001 & $y=0.73 x+4.20$ \\
\hline RBT CL $-0.50 \mathrm{D}$ & 0.44 & 0.0002 & $y=0.74 x+3.35$ \\
\hline RBT CL +5.00 D & 0.32 & 0.0028 & $y=0.68 x+5.52$ \\
\hline Tono-Pen XL & 0.51 & $<0.0001$ & $y=0.59 x+9.54$ \\
\hline Tono-Pen XL CL -5.00 D & 0.32 & 0.0027 & $y=0.37 x+11.3$ \\
\hline Tono-Pen XL CL -0.50 D & 0.09 & 0.1288 & $y=0.23 x+13.1$ \\
\hline Tono-Pen XL CL +5.00 D & 0.35 & 0.0014 & $y=0.71 x+8.78$ \\
\hline
\end{tabular}

Abbreviations: NCT, non-contact tonometer; GAT, Goldmann applanation tonometer; $\mathrm{RBT}$, iCare rebound tonometer; $\mathrm{CL}$, contact lens; $\mathrm{D}$, diopter. 
lenses ( $r=0.46$ and $r=0.44$ respectively). Other comparisons revealed weak correlations. All correlations were significant, except for that observed for the Tono-Pen XL measurements obtained while wearing -0.50 D SCLs.

Using Dunnett's test, we compared the baseline data obtained using the GAT with those obtained using each of the tonometric devices. None of the tonometric devices yielded data that were significantly different from those obtained using the GAT.

As the central cornea became thicker, the IOP measured with all tonometers tended to become higher. This constituted a significant difference when we measured the IOP using the NCT, the RBT, and the GAT. On the other hand, when the data were analyzed using regression analysis, there were no significant differences between spherical equivalent and the IOP measurements using each tonometer.

We examined the correlation of corneal central thickness with the IOP differentials within the devices. There were no significant differences with regard to correlation.

We analyzed the regression of IOP differences from GAT-measured IOP. As GAT-measured IOP increased, IOP differences tended to become larger. The IOP differences from GAT-measured IOP were significant when we measured IOP with the NCT while the subjects were wearing $-5.00 \mathrm{D},-0.50 \mathrm{D}$, or +5.00 D SCLs, GAT while the subjects were wearing $-5.00 \mathrm{D}$ or $-0.50 \mathrm{D}$ SCLs, and TonoPen XL while the subjects were wearing $-5.00 \mathrm{D}$ or $-0.50 \mathrm{D}$ SCLs. Conversely, there were no significant differences in this regard when we measured the IOP using the RBT.

Actually, measuring the IOP using the GAT without SCLs is ideal. However, it is sometimes difficult to do so in an everyday medical examination. Rather, we are sometimes forced to measure the IOP using a limited tonometer combined with SCLs. Therefore, we need to calculate the accuracy of the IOP under non-ideal conditions. The GAT is the gold standard. Therefore, we analyzed the data using BlandAltman plots, while setting the GAT as the standard.

\section{Discussion}

Our results showed no significant differences in the IOP values obtained while wearing negative lenses using NCT, GAT, and RBT compared to the standard IOP values (the IOP values of the naked eyes measured using GAT), except for the GAT measurement with -5.00 D lenses.

Previous studies regarding NCT have demonstrated a small effect of negative lenses, especially when the lens thickness is less than $0.15 \mathrm{~mm} .{ }^{2,4}$ The thickness of the CLs used in our study was $0.084 \mathrm{~mm}$ for the $-5.00 \mathrm{D}$ lenses, 0.124 $\mathrm{mm}$ for the $-0.50 \mathrm{D}$ lenses, and $0.21 \mathrm{~mm}$ for the $+5 \mathrm{D}$ lenses (information obtained from Johnson \& Johnson K.K. Vision Care, Tokyo, Japan). Patel and Illahi ${ }^{3}$ evaluated the IOP values obtained while wearing CLs with power ranging from -15.00 $\mathrm{D}$ to $+13.00 \mathrm{D}$ and concluded that NCT can be performed through a contact lens if the power is not greater than $+3 \mathrm{D}$. In that study, the IOP readings increased in association with the use of thicker hyperopic lenses. ${ }^{1-3}$ Our results are in agreement with these results. Previous studies have also found that the IOP measured using NCT through SCLs is altered depending on the lens power, curvature, thickness, and rigidity. ${ }^{12-14}$ Rimayanti et $\mathrm{al}^{15}$ indicated that SCL use changes the ocular surface behavior and IOP readings during NCT. The radius of curvature of the eye affects the ocular surface displacement and IOP readings. The changes in the curvature of the ocular surface may be responsible for changes in the IOP readings by NCT while wearing SCLs.

In addition, we found no significant differences in the IOP measurements obtained while wearing SCLs using RBT. The Bland-Altman plots also showed that the average IOP difference measured on RBT was close to 0 for all SCL examinations. Zeri et al found no significant differences in the IOP measurements obtained through silicone hydrogel CLs, although significantly lower IOP results were observed while wearing hydrogel CLs of $+2.00 \mathrm{D},-2.00 \mathrm{D}$, and $-6.00 \mathrm{D} .{ }^{9}$ The authors noted that, although the differences were significant, the clinical significance was minimal. RBT bounces a magnetized probe off the cornea, subsequently measuring the deceleration of the probe and converting it into a pressure value. ${ }^{16}$ Zeri et al suggested that the possible decrease in the value of Young modulus of the cornea-CL new body observed in their study accounted for the decrease in the tonometric values obtained without CLs. ${ }^{9}$ The Young's modulus of hydrogel SCLs used in our study is very similar to that of the human cornea; Young's modulus of the human eye has been reported to be $0.29 \pm 0.06$ $\mathrm{Mpa},{ }^{17}$ while that of hydrogel SCLs is $0.29 \pm 0.03 \mathrm{Mpa}$ (information obtained from Johnson \& Johnson K.K. Vision Care, Tokyo, Japan). This may be one reason why our IOP readings obtained through CLs were close to the standard IOP values.

With respect to the IOP measurements obtained with the Tono-Pen XL, we found increased IOP values among the subjects wearing $-5.00 \mathrm{D},-0.50 \mathrm{D}$, and +5.00 D CLs. Previous studies using the Tono-Pen XL found no significant differences regardless of whether the measurements were obtained through plano CLs, ${ }^{7}$ whereas overestimation of the IOP values was observed if performed over high-power $(-3.25 \mathrm{D}$ to -6.00 D) silicon hydrogel CLs. ${ }^{8}$ In the present study, the Tono-Pen XL measurements were significantly higher for all SCLs 
than those obtained in the naked eyes using GAT. The IOP obtained using the Tono-Pen XL is inferred from the pressure on the eyeball with a contact area of $3 \mathrm{~mm}$ diameter between the eyeball and tip of the Tono-Pen XL probe. In order to mediate the measurement obtained over SCLs, the diameter of the eyeball receiving pressure from the Tono-Pen XL may become larger than $3 \mathrm{~mm}$. A larger contact area makes the cornea harder to deform, resulting in a greater force and subsequently larger IOP measurements.

There are several limitations associated with this study. In Table 3, most results of the regression analysis did not show high correlation. This study had a small sample size, and the investigation was performed using only young Japanese subjects. For this reason, we were unable to assess the influence of age or ethnicity on IOP. In addition, we do not know whether our results apply to patients with glaucoma or corneal abnormalities, as we examined only normal eyes. We measured the IOP of young subjects in order to exclude the effects of age on IOP differences. We hope to examine the findings for different age groups in the future.

\section{Conclusion}

We compared the effects of wearing SCLs on the IOP measurements obtained using various instruments. The IOP measurements obtained while wearing +5.00 D lenses were higher than those of the naked eyes measured using GAT, with the exception of the measurements obtained using RBT. The measurements obtained using RBT were similar to those acquired over SCLs ranging from -5.00 D to +5.00 D and those obtained with GAT in the naked eyes. The IOP values obtained while wearing SCLs using NCT exhibited the highest correlation with those obtained using GAT. The NCT and RBT are best when measuring IOP through hydrogel SCLs.

\section{Disclosure}

The authors report no conflicts of interest in this work.

\section{References}

1. Insler MS, Robbin RG. Intraocular pressure by noncontact tonometry with and without soft contact lenses. Arch Ophthalmol. 1987; 105(10):1358-1359.

2. Patel S, Stevenson G. Influence of lens material and intra-ocular pressure on the outcome of non-contact tonometry over soft contact lenses. Cont Lens Anterior Eye. 2009;32(2):68-72.

3. Patel S, Illahi W. Non-contact tonometry over soft contact lenses: effect of contact lens power on the measurement of intra-ocular pressure. Cont Lens Anterior Eye. 2004;27(1):33-37.

4. McMonnies CW. Noncontact tonometry through soft contact lenses. Am J Optom Physiol Opt. 1986;63(12):948-951.

5. Zeri F, Lupelli L, Formichella P, Masci C, Fletcher R. Goldmann applanation tonometry over daily disposable contact lens: accuracy and safety of procedure. Cont Lens Anterior Eye. 2007;30(4):233-238.

6. Allen RJ, Dev Borman A, Saleh GM. Applanation tonometry in silicone hydrogel contact lens wearers. Cont Lens Anterior Eye. 2007; 30(5):267-269.

7. Panek WC, Boothe WA, Lee DA, Zemplenyi E, Pettit TH. Intraocular pressure measurement with the Tono-Pen through soft contact lenses. Am J Ophthalmol. 1990;109(1):62-65.

8. Schornack M, Rice M, Hodge D. Tonopen XL assessment of intraocular pressure through silicone hydrogel contact lenses. Eye Contact Lens. 2012; 38(5):270-273.

9. Zeri F, Calcatelli P, Donini B, Lupelli L, Zarrilli L, Swann PG. The effect of hydrogel and silicone hydrogel contact lenses on the measurement of intraocular pressure with rebound tonometry. Cont Lens Anterior Eye. 2011;34(6):260-265.

10. Firat PG, Cankaya C, Doganay S, et al. The influence of soft contact lenses on the intraocular pressure measurement. Eye (Lond). 2012;26(2):278-282.

11. Gaton DD, Ehrenberg M, Lusky M, et al. Effect of Repeated Applanation Tonometry on the Accuracy of Intraocular Pressure Measurements. Curr Eye Res. 2010;35(6):475-479.

12. Mark HH. Corneal curvature in applanation tonometry. Am JOphthalmol. 1973;76(2):223-224.

13. Ogbuehi KC. The influence of lens power and center thickness on the intraocular pressure measured through soft lenses: a comparison of two noncontact tonometers. Cont Lens Anterior Eye. 2012; 35(3):118-128.

14. Liu YC, Huang JY, Wang IJ, Hu FR, Hou YC. Intraocular pressure measurement with the noncontact tonometer through soft contact lenses. J Glaucoma. 2011;20(3):179-182.

15. Rimayanti U, Kiuchi Y, Uemura S, Takenaka J, Mochizuki H, Kaneko M. Ocular Surface Displacement with and without Contact Lenses during Non-Contact Tonometry. PLoS One. 2014; 9(4):e96066.

16. Kontiola A. A new electromechanical method for measuring intraocular pressure. Doc Ophthalmol. 1997;93(3):265-276.

17. Hamilton KE, Pye DC. Young's modulus in normal corneas and the effect on applanation tonometry. Optom Vis Sci. 2008;85(6):445-450.
Clinical Ophthalmology

\section{Publish your work in this journal}

Clinical Ophthalmology is an international, peer-reviewed journal covering all subspecialties within ophthalmology. Key topics include: Optometry; Visual science; Pharmacology and drug therapy in eye diseases; Basic Sciences; Primary and Secondary eye care; Patient Safety and Quality of Care Improvements. This journal is indexed on Submit your manuscript here: http://www.dovepress.com/clinical-ophthalmology-journal

\section{Dovepress}

PubMed Central and CAS, and is the official journal of The Society of Clinical Ophthalmology (SCO). The manuscript management system is completely online and includes a very quick and fair peer-review system, which is all easy to use. Visit http://www.dovepress.com/ testimonials.php to read real quotes from published authors. 